\title{
Are risk averse agents more optimistic? A Bayesian estimation approach
}

\author{
Selima Ben Mansour, Elyès Jouinił Jean-Michel Marin, Clotilde Napp* \\ and Christian Robert ${ }^{\dagger}$
}

\begin{abstract}
Our aim is to analyze the link between optimism and risk aversion in a subjective expected utility setting and to estimate the average level of optimism when weighted by risk tolerance. This quantity is of particular importance since it characterizes the consensus belief in risk-taking situations with heterogeneous beliefs. Its estimation leads to a nontrivial statistical problem. We start from a large lottery survey (1536 individuals). We assume that individuals have true unobservable characteristics and that their answers in the survey are noisy realizations of these characteristics. We adopt a Bayesian approach for the statistical analysis of this problem and use an hybrid MCMC approximation method to numerically estimate the distributions of the unobservable characteristics. We obtain that individuals are on average pessimistic and that pessimism and risk tolerance are positively correlated. As a consequence, we conclude that the consensus belief is biased towards pessimism.

Keywords: Bayesian estimation, MCMC scheme, importance sampling, pessimism, risk tolerance, risk aversion, consensus belief.
\end{abstract}

\section{Introduction}

Optimism is usually conceived as a personal trait leading to overestimate the likelihood of good outcomes and to underestimate the likelihood of bad outcomes. Previous work related to this issue include psychological surveys as well as empirical studies on professionals' economic forecasts (see, e.g., Fried and Givoly, 1982, O'Brien, 1988, Francis and Philbrick, 1993, Kang et al., 1994, Dreman and Berry, 1995, Giordani and Söderlind, 2005).

Risk-aversion in decision making intuitively corresponds to the fact that when facing choices with comparable returns, agents tend to choose the less risky alternative. This feature has been largely studied in particular in relation with other personal characteristics like gender, age, income, education (see, among others, Binswanger, 1980, 1981, Palson, 1996, Barsky et al., 1997, Eisenhauer and Halek, 2001, Eisenhauer and Ventura, 2003, Felton et al., 2003, Guiso and Paiella, 2001).

In the setting of a Subjective Expected Utility (SEU) model, risk aversion is measured by the curvature of the utility function and optimism is characterized by the individual subjective probability. Risk aversion and individual subjective probability completely determine the individual

\footnotetext{
${ }^{*}$ DRM, UMR-CNRS 7088, Université Paris-Dauphine

${ }^{\dagger}$ CEREMADE, UMR-CNRS 7534, Université Paris-Dauphine.

${ }^{\ddagger}$ Corresponding author: jouini@ceremade.dauphine.fr, phone: +331440542 26, fax: +33144054849.

$\S$ INRIA Futurs, Projet SELECT, Université Paris-Sud
} 
decision-making behavior, at least locally. The SEU model is particularly well adapted to the aggregation problem and recent papers show how individual risk aversions and subjective probabilities are aggregated at the collective level in such a setting (Calvet et al. 2002, Gollier, 2003, Jouini and Napp, 2006, 2007, Li, 2006).

From an empirical point of view, these two notions are hard to disentangle. For instance, in the context of lotteries, which is often used to measure the risk aversion level, the reservation price of a risk-averse (resp. risk tolerant) and unbiased individual is empirically indistinguishable from the reservation price of a risk-neutral and pessimistic (resp. optimistic) individual; there is here an identification problem that confounds data interpretation.

In this paper, we start from a lottery survey, in which data on both risk aversion (reservation price) and subjective beliefs (subjective probabilities) is available in the same context. In the setting of a SEU model, this survey enables us to disentangle both notions and to infer about the individual levels of optimism and of risk aversion. Our aim is then to determine and to understand the nature of the link between optimism and risk aversion. More precisely, we want to estimate the average level of optimism weighted by the risk tolerance ${ }^{1}$ as well as the unweighted average.

The estimation of these quantities is important for both following reasons. First, since the difference between the weighted average and the unweighted average of the individual level of optimism is precisely given by the covariance between risk tolerance and optimism, the estimation of these averages will give us the nature of the correlation between risk tolerance and optimism. The determination of this correlation should shed some light on the determinants of the level of optimism and/or risk aversion at the individual level. Second, the estimation of the weighted average of the individual beliefs is of particular importance at the collective level. Indeed, in the setting of collective risk-taking decisions with heterogeneous beliefs (and SEU), this average subjective belief represents the consensus of the group (see Gollier, 2003). Analogously, in CCAPM ${ }^{2}$-like equilibrium models with heterogeneous beliefs (and SEU), the weighted average of the individual beliefs ${ }^{3}$ represents the belief of the representative agent (see Rubinstein, 1976, Detemple and Murthy, 1994, Calvet et al., 2002, Abel, 1989, Zapatero, 1998, Jouini and Napp, 2006, 2007, Li, 2006). This consensus belief plays a crucial role on the Pareto optimal risk sharing rules and on the equilibrium properties. In particular, a pessimistic consensus belief might help in explaining the risk premium and risk-free rate puzzles ${ }^{4}$.

We analyze answers to a large survey (1536 individuals). The main problem we encounter in this survey is related to the fact that some observations correspond to risk-neutral or even risk-seeking behaviour. These observations are not compatible with the standard assumptions of decision theory and of CCAPM-like equilibrium models. Moreover, for these individuals, the risk tolerance level is infinite or negative, and with such infinite or negative weights, an average weighted by the risk tolerance becomes meaningless. It is not possible to simply reject these observations, since they are associated with high levels of risk tolerance and their rejection would have a strong impact on the quantities to be estimated. Note that the presence of risk-seeking or risk-neutral individuals in the panel is not surprising. Similar results have been obtained in the literature (Guiso and Paiella, 2001, Eisenhauer and Ventura, 2003, Hartog et al., 2002). However, our aim is to provide a proxy for risk aversion in real life situations and it is natural to assume that this "real life" risk aversion is positive.

\footnotetext{
${ }^{1}$ Risk tolerance is defined as the inverse of risk aversion.

${ }^{2}$ Consumption-based Capital Asset Pricing Model.

${ }^{3}$ When agents are assumed to have log utilities, this corresponds to the wealth weighted average belief.

${ }^{4}$ As shown by Mehra and Prescott (1985) and Weil (1989), standard models of financial markets equilibrium predict risk-premia (resp. risk-free rates) that are too low (resp. too high) to fit observed data.
} 
For all these reasons, these observations require a specific treatment. We propose to interpret both risk neutrality and risk seeking as noise and we adopt the following approach; we assume that individuals have true unobservable characteristics that respect the assumptions of decision theory, and we interpret the answers in the survey as noisy realizations of those characteristics.

The aim is then to estimate the distribution of the unobservable characteristics and to derive the value of the average level of optimism weighted by the risk tolerance. We follow a Bayesian approach for this purpose and use an MCMC approximation method for computational reasons. We emphasize that we do not pretend to compute a precise level of optimism of the consensus belief in a general setting but rather to determine whether there is a clear spread between the weighted and unweighted averages, which would indicate a behavioral correlation between optimism and risk aversion and a bias at the aggregate level.

The paper is organized as follows. Section 2 presents the data as well as the measures of individual optimism and risk aversion. Section 3 presents the statistical estimation. Section 4 concludes.

\section{The data}

We first describe the survey, then we present the adopted risk aversion and optimism measures. The last subsection deals with data consistency results.

\subsection{The survey instrument}

We start from answers to a survey conducted face-to-face in the field by professional interviewers. The sample is made of adults, between 22 and 55, that are randomly chosen, and yielded 1,536 responses. The questionnaire consists of essentially five parts ${ }^{5}$.

In the first part, the participant is asked about his willingness to take part in a game of chance. Only the individuals who are willing to take part in a game of chance go on with the questionnaire.

In the second part, the participant is offered the opportunity of entering a head and tail game for ten rounds. More precisely, a coin is being flipped ten times; each time head occurs, the participant is supposed to get 10 Euros. The participant is then asked for his own estimation of the number of times head will occur. The participant is also asked to reveal the maximum amount he is willing to pay in order to take part in this game. The aim of this second part is to obtain, in the same setting, measures of the individual levels of optimism and of risk aversion.

The third part deals with questions similar to those in Barsky et al. (1997) which lead to the elicitation of the level of individual relative risk aversion. This part will enable us to verify that the data on absolute risk aversion is consistent with the data on relative risk aversion at the individual level. The fourth part deals with optimism questions as in Wenglert and Rosen (2000) while the fifth part deals with personal questions, such as gender, age, marital status, employment status, education and income.

These last three parts will make it possible to compare the results obtained on our sample with those usually obtained in the literature in terms of risk-aversion, optimism and their determinants.

\subsection{Risk-aversion and optimism measures}

We derive individual measures of risk aversion and of optimism from the answers to the survey.

\footnotetext{
${ }^{5}$ It is available at http://www.ceremade.dauphine.fr/jouini.
} 
As in Ben Mansour et al. (2006), we derive a measure of the degree of optimism from the answers to the first question of the second part of the questionnaire "Imagine that a coin will be flipped 10 times. Each time, if head, you win $10 €$. What is your own estimation, according to your experience and your luck, of the number of times head will occur, i.e. how many times (out of ten) do you think you are going to win (and get $10 €$ )?" This measure of optimism ${ }^{6}$ is directly given by the number of times $x_{i}$ that the individual $i$ thinks he is going to win. An optimistic individual is characterized by a value of $x_{i}$ above the objective value of 5 and the distance to 5 measures the intensity of his optimism.

This measure is obviously compatible with the definition of optimism as a transformation of the objective distribution into a more favorable one. Indeed, the subjective probability of individual $i$ associated to the favourable event "head occurs" is approximated by $\pi_{i}=\frac{x_{i}}{10}$ and an optimistic individual is characterized by a $\pi_{i}$ strictly higher than 0.5 . We will use this measure of individual subjective probability when determining a measure of risk aversion in the framework of Subjective Expected Utility. Notice that the introduction of a ten times head-tail game is intended to provide the individuals with a simple framework, helping them to reveal their subjective probability associated with the event "head occurs".

As far as risk aversion is concerned, we recall that absolute risk aversion is defined by the quantity $A(c)=-\frac{u^{\prime \prime}(c)}{u^{\prime}(c)}$, whereas relative risk aversion is defined by $R(c)=-c \frac{u^{\prime \prime}(c)}{u^{\prime}(c)}$, where $u$ denotes the agent's utility function. Adopting an approach similar to Guiso and Paiella (2001), we will use the answers to the question "What is the maximum amount that you are willing to pay to take part in the game?" to derive a measure of the individual level of absolute risk aversion. The answer of individual $i$, denoted by $y_{i}$, corresponds to his reservation price, which leaves him indifferent between his initial wealth and the expected gain from the game. This means that if we adopt the SEU model and if we let $\widetilde{X}$ denote the random payoff associated with the head-tail game, the answer $y_{i}$ satisfies

$$
\begin{aligned}
u_{i}\left(w_{i}\right) & =E^{i}\left[u_{i}\left(w_{i}+\widetilde{X}-y_{i}\right)\right] \\
& =\sum_{k=0}^{10} C_{10}^{k} \pi_{i}^{k}\left(1-\pi_{i}\right)^{10-k} u_{i}\left(w_{i}+10 k-y_{i}\right),
\end{aligned}
$$

where $u_{i}$ denotes the utility function of agent $i, w_{i}$ his initial wealth and $E^{i}$ the expectation operator under his subjective probability. In the case of exponential utility functions, a measure for (corrected) absolute risk aversion of individual $i$ is then given by the solution $a_{i}$ of

$$
1=\sum_{k=0}^{10} C_{10}^{k} \pi_{i}^{k}\left(1-\pi_{i}\right)^{10-k} \exp a_{i}\left(y_{i}-10 k\right) .
$$

It is easy to obtain through a simple factorization that this equation is equivalent to

$$
1=\pi_{i} \exp a_{i}\left(\frac{y_{i}}{10}-10\right)+\left(1-\pi_{i}\right) \exp a_{i} \frac{y_{i}}{10} .
$$

We denote by $C A_{i}$ the solution, obtained by numerical approximation, of Equation (1). We refer to it as Corrected absolute risk Aversion because, unlike previous experimental or empirical literature on risk aversion in an expected utility framework, we take into account the subjective probability

\footnotetext{
${ }^{6}$ Ben Mansour et al. (2006) provide a rationale for this measure as well as an analysis of its main features.
} 
$\pi_{i}$ in order to determine the individual level of risk aversion. For instance, in Guiso and Paiella (2001), when the individual is told to have a chance of 1 over 10 of winning, one does not take into account, both in the interpretations of the results and in the inferred levels of risk aversion, the fact that some people believe that they are lucky and overweight their probability of winning while others do the opposite. The same remark holds for empirical approaches. For instance, Eisenhauer and Halek (2001) estimate the level of risk aversion using life insurance data. The underlying model assumes an age based estimated probability of mortality, i.e. assumes that all people in the same age class share the same objective estimation of their probability of mortality, whereas it is intuitive that the estimation of one's probability of mortality is subjective and highly dependent upon one's optimism.

\subsection{Consistency of the data}

In this section we verify the consistency of our data from different points of view.

First, the answers of our sample seem consistent with previous surveys. As far as relarive risk aversion is concerned, we find that women are more risk averse (as in Eisenhauer and Halek, 2001, Barsky et al., 1997, Cohn et al., 1975, Palsson, 1996, Jianakoplos and Bernasek, 1998, Hariharan et al., 2000) and that relative risk aversion increases with age (as in McInish, 1982, Morin and Suarez, 1983, Riley and Chow, 1992, Palsson, 1996, Schooley and Worden, 1996, Wang and Hanna, 1997, Barsky et al., 1997).

The answers about optimism in the fourth part are also consistent with previous surveys. We obtain on our sample the same results as Wenglert and Rosen (2000) about personal optimism. Indeed, Wenglert and Rosen (2000) obtain an average level of "personal optimism" equal to 0.596 and we obtain a value of 0.536 (using the same methodology).

The consistency with previous surveys is also confirmed by the data on reservation prices. Let us denote by $O A_{i}$, i.e. the solution of Equation (1) for $\pi_{i}=\frac{1}{2}$, i.e. the solution $a_{i}$ of

$$
1=\frac{1}{2} \exp a_{i}\left(\frac{y_{i}}{10}-10\right)+\frac{1}{2} \exp a_{i} \frac{y_{i}}{10} .
$$

In other words, $O A$ corresponds to the absolute risk aversion under the objective probability as it is usually computed in the literature. We obtain that $O A$ is higher for women, increases with age and decreases with income as in Guiso and Paiella (2001), Donkers et al. (2001), Hartog et al (2002), Powell and Ansic (1997), Eisenhauer and Ventura (2003).

A possible drawback of our approach is that we use essentially two simple questions only to elicit individual risk attitudes. In fact, the survey contains various questions that involve risk aversion or optimism. In order to elicit one of these characteristics, the classical procedure would be a regression based on all the questions related to that characteristic. However, our aim is to determine both characteristics in the same context in order to disentangle the two notions and to obtain a measure of risk aversion that is corrected by the level of optimism.

For this reason, we have chosen to determine a measure of the individual levels of optimism and risk aversion from the answers to the head and tail questions only and then to verify their the answers to other questions in the survey.

We verify that the question on the reservation price are compatible with those on relative risk aversion. By definition, relative risk aversion is equal to the product of absolute risk aversion by individual wealth. We consider individual income as a proxy for individual wealth. Since relative risk aversion is a qualitative variable (individuals are sorted into seven groups), we establish the 
consistency between relative and absolute risk aversion measures through a comonotonicity test between relative risk aversion and the product of absolute risk aversion by the mean of the income band (see Table 1).

Finally, as far as the data on the subjective probabilities are concerned, three questions in the questionnaire on personal pessimism have attracted our attention since intuitively, they should have a link with our notion of pessimism : (Q1) You have no chance to win at a lottery game, (Q2) You have no chance to be selected for a television game and (Q3) You will win one day at the Promosport. As expected, we have obtained a decreasing relationship between (Q1) (resp. Q2) and our measure of optimism and an increasing relationship between (Q3) and our measure of optimism.

\section{Statistical estimation}

In this section we adopt a Bayesian approach (see, e.g., Robert, 2001) for the estimation of the average of the subjective probabilities weighted by the risk tolerance. We also estimate the unweighted average of the subjective probabilities.

The estimation of these quantities is interesting at the individual level, and can help to understand the nature of the link between risk aversion and optimism. Indeed, the correlation between risk aversion and optimism is easily deduced from the difference between these two quantities.

Moreover, the estimation of the weighted and unweighted averages of the individual beliefs is of particular importance at the collective level. Indeed, in the setting of collective risk-taking decisions with heterogeneous beliefs (and SEU), this average subjective belief represents the consensus of the group. Analogously, in CCAPM-like equilibrium models with heterogeneous beliefs (and SEU), the weighted average of the individual beliefs represents the belief of the representative agent. The determination of the consensus belief, or more precisely of the extent to which it differs from the unweighted average of the subjective beliefs, is then particularly interesting.

We do not pretend here to be able to compute precise and reliable individual and collective levels of risk aversion or of optimism. We are interested in observing whether there is a clear spread between the weighted and unweighted averages, which would indicate a behavioral correlation between optimism and risk aversion and a bias at the aggregate level. In particular, it is wellknown that willingness-to-pay questions are biased but this bias should not induce by itself a correlation between optimism and risk aversion.

\subsection{Descriptive statistics}

The results on the measure of optimism introduced in Section 2 have been studied in Ben Mansour et al. (2006). We recall here the main features of this analysis. Figure 1 provides the distribution of our measure of optimism among individuals and shows that there is a great heterogeneity in the level of optimism among agents. For the considered sample of 1,536 individuals, the mean value for the number of times the individual thinks he is going to win is equal to 3.93. It is significant and quite striking to observe that when asked about the number of times he is going to win at a head and tail game for ten draws, an average individual does not answer 5 times as he should if thinking under the objective probability, but answers slightly less than 4 times. This result is in favor of the existence of a behavioral bias towards pessimism in individual beliefs.

Regarding risk aversion, the average announced price is $11.96 €$ which, as expected, is lower 
than the average subjectively expected payoff. However some agents announced a reservation price higher than their subjectively expected gain hence have a negative corrected risk aversion. This can be shown if we focus on the probabilities and prices that we denoted respectively by $x_{i}$ and $y_{i}$ and if we represent them on a diagram. Figure 2 contains a jitter ${ }^{7}$ representation of this diagram. There are 55 points such that $y / 100=x / 10$ and 40 points such that $y / 100>x / 10$.

Based on Figures 2, it appears that there is a negative link between non corrected risk aversion and optimism, leading us to conclude that risk aversion (in the usual sense) decreases with optimism. This correlation is confirmed as statistically significant by Pearson, Kendall and Spearman tests. This is clearly understandable since the more optimistic an individual, the lower his non-corrected risk aversion. In other words, the non-corrected measure of risk aversion contents a great dose of pessimism. This negative correlation does not hold for corrected risk aversion. On the contrary, we verified through Spearman's and Kendall's tests that the average of the corrected risk aversion for a given level of optimism significantly increases with the level of optimism (see Table 2). This is in favor of a positive correlation between risk aversion (in our sense) and optimism.

\subsection{The Bayesian model}

We now want to estimate the average level of optimism weighted by the risk tolerance. The problem is that there is a non negligible number of individuals for which $y / 100=x / 10$ (i.e. zero corrected risk aversion and infinite corrected risk tolerance) or even $y / 100>x / 10$ (i.e. negative corrected risk aversion and risk tolerance). The presence of such individuals raises some specific problems since with such negative or infinite weights, the weighted sum is not a weighted average any longer. Furthermore, we can not simply eliminate these observations; indeed, the individuals for which $y / 100$ is near $x / 10$ are those for which the corrected risk tolerance level is high, hence they are heavily weighted in the considered average. Finally, such observations are incompatible with the classical assumptions of decision theory and CCAPM-like equilibrium models.

For all these reasons, a specific treatment of the data needs to be undertaken. One should bear in mind the fact that any slight modification of an observation such that $y / 100$ is near $x / 10$ has a strong impact on the risk tolerance level and then on the weight in the considered average.

Note that the presence of risk-seeking or risk-neutral individuals in the panel is not surprising. Similar results have been obtained in the literature (Guiso and Paiella, 2001, Eisenhauer and Ventura, 2003, Hartog et al., 2002). Moreover, the amount considered in this study is relatively small (100 Euros versus 0 Euro), so that one cannot expect to find much concavity in the utility function. In other words, it is not surprising to find risk-seeking behaviors for such small amounts. However, our aim is to provide a proxy for the risk aversion in real life situations and it is natural to assume that this "real life" risk aversion is positive. Furthermore, at the equilibrium and at least locally, the agents are necessarily risk averse. For this reason, we propose to interpret both risk neutrality and risk seeking as resulting from noise.

More precisely, we adopt the following approach. We assume that individuals have true unobservable characteristics $\left(\pi_{i}, p_{i}\right)$, where $\pi_{i}$ corresponds to the subjective probability of success at each draw and $p_{i}$ corresponds to the price that the individual is willing to pay for the ten draw game normalized to one (i.e. divided by 100). The answer $\left(x_{i}, y_{i}\right)$ is then interpreted as a noisy realization of $\left(\pi_{i}, p_{i}\right)$.

\footnotetext{
${ }^{7}$ The jitter representation is used to improve the rendering on samples on grids by introducing a slight perturbation that exhibits the number of points at each location on the grid.
} 
More precisely, we assume that $(\mathbf{x}, \mathbf{y})=\left(\left(x_{1}, y_{1}\right), \ldots,\left(x_{n}, y_{n}\right)\right)$ is a set of independent realizations such that

$$
\left(x_{i}, y_{i}\right) \mid\left(\pi_{i}, p_{i}\right) \sim \mathscr{B}\left(10, \pi_{i}\right) \otimes \mathscr{B}\left(100, p_{i}\right)
$$

where $n$ is equal to 1536 .

We now follow a Bayesian strategy to infer about the parameter $\left(\pi_{i}, p_{i}\right)$, first defining a prior distribution on this parameter as

$$
f_{(\pi, p)}\left(\pi_{i}, p_{i}\right)=\frac{\pi_{i}^{a-1}\left(1-\pi_{i}\right)^{b-1} p_{i}^{c-1}\left(1-p_{i}\right)^{d-1}\left(\pi_{i}-p_{i}\right)^{e}}{g(a, b, c, d, e)} \mathbb{I}_{0<p_{i}<\pi_{i}<1}
$$

where $a, b, c, d, e$ are positive constants and

$$
\begin{aligned}
g(a, b, c, d, e) & =B(a+c+e, b) B(c, e+1) \\
& +\sum_{i=1}^{\infty} \frac{(-d+1) \times \ldots \times(-d+i)}{i !} B(a+c+e+i, b) B(c+i, e+1) .
\end{aligned}
$$

is the normalizing constant of the probability density (2).

We have then

$$
\begin{gathered}
f\left(\left(x_{1}, y_{1}, \pi_{1}, p_{1}\right), \ldots,\left(x_{n}, y_{n}, \pi_{n}, p_{n}\right) \mid a, b, c, d, e\right)= \\
\prod_{i=1}^{n}\left[C_{10}^{x_{i}} \pi_{i}^{x_{i}}\left(1-\pi_{i}\right)^{10-x_{i}} \mathbb{I}_{\{0, \ldots, 10\}}\left(x_{i}\right) C_{100}^{y_{i}} p_{i}^{y_{i}}\left(1-p_{i}\right)^{100-y_{i}} \mathbb{I}_{\{0, \ldots, 100\}}\left(y_{i}\right)\right. \\
\left.\frac{\pi_{i}^{a-1}\left(1-\pi_{i}\right)^{b-1} p_{i}^{c-1}\left(1-p_{i}\right)^{d-1}\left(\pi_{i}-p_{i}\right)^{e}}{g(a, b, c, d, e)} \mathbb{I}_{] 0,1[}\left(\pi_{i}\right) \mathbb{I}_{] 0, \pi_{i}[}\left(p_{i}\right)\right]
\end{gathered}
$$

which is a joint distribution on the data $(\mathbf{x}, \mathbf{y})$ and on the parameter. The Bayesian estimation is based on the so-called posterior distribution of the parameter, which simply is the conditional distribution of this parameter given the data, $f_{(\pi, p)}\left(\pi_{1}, p_{1}, \ldots, \pi_{n}, p_{n} \mid \mathbf{x}, \mathbf{y}, a, \cdots, e\right)$.

The sample $(\pi, \mathbf{p})=\left(\left(\pi_{1}, p_{1}\right), \ldots,\left(\pi_{n}, p_{n}\right)\right)$ is not observed and, in addition, we first assume that the so-called hyperparameters $a, b, c, d, e$ are also unknown and thus need to be estimated from the data ( $\mathbf{x}, \mathbf{y})$ (using similar Bayesian principles).

Once $(a, b, c, d, e)$ is estimated, we want to estimate the average subjective probability weighted by the risk tolerance as well as the unweighted average subjective probability. For a given pair $(\pi, p)$, the associated level of risk aversion $z(\pi, p)$ is defined as the solution in $z$ of

$$
\pi \exp (z(p-1))+(1-\pi) \exp (z p)-1=0
$$

which is the analog of Equation (1). Hence, based on the data $(\mathbf{x}, \mathbf{y})$, we want to estimate the ratio $\mathbb{E}[\pi / z(\pi, p)] / \mathbb{E}[1 / z(\pi, p)]$, which is given by

$$
\mathcal{I}=\frac{\int_{0}^{1}\left(\int_{0}^{u}\left[\frac{u}{z(u, v)}\right] \frac{u^{a-1}(1-u)^{b-1} v^{c-1}(1-v)^{d-1}(u-v)^{e}}{g(a, b, c, d, e)} d v\right) d u}{\int_{0}^{1}\left(\int_{0}^{u}\left[\frac{1}{z(u, v)}\right] \frac{u^{a-1}(1-u)^{b-1} v^{c-1}(1-v)^{d-1}(u-v)^{e}}{g(a, b, c, d, e)} d v\right) d u}=\frac{\mathcal{I}_{3}}{\mathcal{I}_{4}} .
$$

The natural Bayes estimate of this ratio is then the posterior expectation, $\mathbb{E}\left[\mathcal{I}_{3} / \mathcal{I}_{4} \mid \mathbf{x}, \mathbf{y}\right]$ (see Robert, 2001, Chapter 2). 
The unusual prior distribution for $(\pi, p)$ given in (2) is inspired from Beta distributions that are the conjugates of binomial distributions, the additional term $\left(\pi_{i}-p_{i}\right)^{e}$ being chosen for integrability reasons. Indeed, near the diagonal, we have that $z(\pi, p) \sim \pi-p$ and thus the nature of the integrals $\mathcal{I}_{3}$ and $\mathcal{I}_{4}$ depends on the position of $e$ with respect to zero.

We will also estimate the unweighted average level of optimism $\mathcal{I}_{1}$ as well as the unweighted average level of risk aversion $\mathcal{I}_{2}$, respectively given by

$$
\begin{aligned}
& \mathcal{I}_{1}= \frac{\int_{0}^{1}\left(\int_{0}^{u} \frac{u^{a}(1-u)^{b-1} v^{c-1}(1-v)^{d-1}(u-v)^{e}}{g(a, b, c, d, e)} d v\right) d u}{\int_{0}^{1}\left(\int_{0}^{u} \frac{u^{a-1}(1-u)^{b-1} v^{c-1}(1-v)^{d-1}(u-v)^{e}}{g(a, b, c, d, e)} d v\right) d u} \\
& \mathcal{I}_{2}=\frac{\int_{0}^{1}\left(\int_{0}^{u} z(u, v) \frac{u^{a-1}(1-u)^{b-1} v^{c-1}(1-v)^{d-1}(u-v)^{e}}{g(a, b, c, d, e)} d v\right) d u}{\int_{0}^{1}\left(\int_{0}^{u} \frac{u^{a-1}(1-u)^{b-1} v^{c-1}(1-v)^{d-1}(u-v)^{e}}{g(a, b, c, d, e)} d v\right) d u} .
\end{aligned}
$$

\subsection{Parameter estimation and numerical results}

To complete the definition of the prior distribution, we also introduce the following exponential prior on the hyperparameters:

$$
a, b, c, d, e \stackrel{\mathrm{iid}}{\sim} \mathcal{E} x p(1) .
$$

Once the prior and the sampling distributions selected, we can formally draw inference about the true parameters $\left(\pi_{i}, p_{i}\right)$ by computing their posterior distribution, that is, their distribution conditional on the observations, and using this distribution as a complete summary of the information available on the $\left(\pi_{i}, p_{i}\right)$ 's (Robert, 2001). In practice, it is however quite complex to derive exactly the posterior distribution of a pair $\left(\pi_{i}, p_{i}\right)$, given that this derivation implies the integration of all the other parameters conditional on the whole sample $(\mathbf{x}, \mathbf{y})$. We therefore need to resort to an approximate method, based on the simulation of Markov chains, namely the Markov Chain Monte Carlo (MCMC) method (Robert and Casella, 2004). Gibbs sampling is one of the most popular MCMC schemes. Its principle is quite straightforward: when faced with the simulation of a multivariate distribution $g\left(\theta_{1}, \ldots, \theta_{p}\right)(p>1)$, generate instead successively from the full conditionals $g_{i}\left(\theta_{i} \mid \theta_{-i}\right)$ and let the corresponding Markov chain converge to the limiting distribution $\pi(\theta)$. In our case, the implementation of this principle translates into the generation of the $\left(\pi_{i}, p_{i}\right)$ 's given $(a, b, c, d, e)$ and $(\mathbf{x}, \mathbf{y})$, and of $(a, b, c, d, e)$ given the $\left(\pi_{i}, p_{i}\right)$ 's and $(\mathbf{x}, \mathbf{y})$. More precisely, the conditional independence relations imply that we simulate successively from $(1 \leq i \leq n)$

- $f_{(p, \pi)}\left(\pi_{i}, p_{i} \mid \ldots\right) \propto \pi_{i}^{a+x_{i}-1}\left(1-\pi_{i}\right)^{b+10-x_{i}-1} p_{i}^{c+y_{i}-1}\left(1-p_{i}\right)^{d+100-y_{i}-1}\left(\pi_{i}-p_{i}\right)^{e} \mathbb{I}_{0<\pi_{i}<p_{i}<1}$;

- $f_{a}(a \mid \ldots) \propto \frac{1}{g(a, b, c, d, e)^{n}} \exp \left(-\left(1-\sum_{i=1}^{n} \log \left(\pi_{i}\right)\right) a\right) \mathbb{I}_{] 0, \infty[}(a)$;

- $f_{b}(b \mid \ldots) \propto \frac{1}{g(a, b, c, d, e)^{n}} \exp \left(-\left(1-\sum_{i=1}^{n} \log \left(1-\pi_{i}\right)\right) b\right) \mathbb{I}_{0, \infty[}(b)$;

- $f_{c}(c \mid \ldots) \propto \frac{1}{g(a, b, c, d, e)^{n}} \exp \left(-\left(1-\sum_{i=1}^{n} \log \left(p_{i}\right)\right) c\right) \mathbb{I}_{0, \infty[}(c)$;

- $f_{d}(d \mid \ldots) \propto \frac{1}{g(a, b, c, d, e)^{n}} \exp \left(-\left(1-\sum_{i=1}^{n} \log \left(1-p_{i}\right)\right) d\right) \mathbb{I}_{] 0, \infty[}(d)$; 
- $f_{e}(e \mid \ldots) \propto \frac{1}{g(a, b, c, d, e)^{n}} \exp \left(-\left(1-\sum_{i=1}^{n} \log \left(\pi_{i}-p_{i}\right)\right) e\right) \mathbb{I}_{] 0, \infty[}(e)$.

Note that the dataset $(\mathbf{x}, \mathbf{y})$ only appears in the conditional distribution $f_{(p, \pi)}$. This is due to the hierarchical nature of the model that implies that $(a, \ldots, e)$ is independent of $(\mathbf{x}, \mathbf{y})$ given the $\left(\pi_{i}, p_{i}\right)$ 's.

These distributions are moreover far from standard and their simulation requires another level of MCMC technology, namely the replacement of the exact simulation with a Markov step which guarantees convergence to the proper distribution. The corresponding algorithm is called hybrid $M C M C$ and works as described in the Appendix.

We however abstain from reporting the full simulation experiment corresponding to this setting as it provides a non-identifiability conclusion on the parameter $e$. Indeed, the chain is always converging to a value of $e$ very close to 0 . This is particularly worrying because the parameter $e$ has been introduced in order to ensure the finiteness of $\mathcal{I}_{3}$ and $\mathcal{I}_{4}$. A value of $e=0$ would have been more natural for the distribution of $(\pi, p)$ given the choice of binomial distributions for $\left(x_{i}, y_{i}\right) \mid\left(\pi_{i}, p_{i}\right)$. In order to examine this problem, we study instead the sensitivity of the model to changes in $e$. More precisely, we will fix different small values for $e$ hoping that the ratio $\frac{\mathcal{I}_{3}}{\mathcal{I}_{4}}$ will remain stable when $e$ goes to zero even if we know that $\mathcal{I}_{3}$ and $\mathcal{I}_{4}$ will diverge.

When setting the value of $e$ to 0.1,0.05, and 0.01, running an hybrid MCMC algorithm leads to a highly stable estimation of the other parameters. The results of this simulation for $e=0.01$ are presented in Figures 3 and 4 while the Bayesian estimates of $a, b, c, d$ are given in Table 3 for the different chosen values of $e$. The estimates are quite close and the mixing behavior of the MCMC chains seems to be fairly impervious to changes in $e$. We are therefore satisfied with this proxy to the full parameter estimation scheme and in the next we will retain a value of 0.01 for $e$.

Now, for a given sample $(\mathbf{x}, \mathbf{y})$, we want to estimate the quantity

$$
\mathcal{I}=\frac{\int_{0}^{1}\left(\int_{0}^{u}\left[\frac{u}{z(u, v)}\right] \frac{u^{a-1}(1-u)^{b-1} v^{c-1}(1-v)^{d-1}(u-v)^{e}}{g(a, b, c, d, e)} d v\right) d u}{\int_{0}^{1}\left(\int_{0}^{u}\left[\frac{1}{z(u, v)}\right] \frac{u^{a-1}(1-u)^{b-1} v^{c-1}(1-v)^{d-1}(u-v)^{e}}{g(a, b, c, d, e)} d v\right) d u}=\frac{\mathcal{I}_{3}}{\mathcal{I}_{4}} .
$$

Since this ratio depends on the unknown parameters $(a, b, c, d)$, e being fixed, the natural estimator is the Bayes posterior expectation conditional on the sample $(\mathbf{x}, \mathbf{y}), \mathbb{E}[\mathcal{I} \mid(\mathbf{x}, \mathbf{y})]$. There is however a difficulty in that both integrals $\mathcal{I}_{3}$ and $\mathcal{I}_{4}$ are not available in closed form. We thus resort to separate evaluations of both integrals, with $\mathcal{I}_{3}$ and $\mathcal{I}_{4}$ estimated by importance sampling in order to ensure finite variance estimates (Robert and Casella, 2004). The importance distribution we use is a Dirichlet $\mathcal{D}(\psi, e-\epsilon, \lambda)$ with $\psi>0, e-\epsilon>0$ and $\lambda>0$, and density

$$
v_{i}^{\psi-1}\left(u_{i}-v_{i}\right)^{e-\epsilon-1}\left(1-u_{i}\right)^{\lambda-1} \Gamma(\psi+e-\epsilon+\lambda) /(\Gamma(\psi) \Gamma(e-\epsilon) \Gamma(\lambda)),
$$

due to the similarity with the target distribution and the warranty of a finite variance. The importance sampling estimates of both integrals are therefore

$$
\widetilde{\mathcal{I}_{3}}=\frac{1}{N} \sum_{i=1}^{N} \frac{u_{i}^{a_{i}}\left(1-u_{i}\right)^{b_{i}-1} v_{i}^{c_{i}-1}\left(1-v_{i}\right)^{d_{i}-1}\left(u_{i}-v_{i}\right)^{e} / g\left(a_{i}, b_{i}, c_{i}, d_{i}, e\right)}{z\left(u_{i}, v_{i}\right) v_{i}^{\psi-1}\left(u_{i}-v_{i}\right)^{e-\epsilon-1}\left(1-u_{i}\right)^{\lambda-1} \Gamma(\psi+e-\epsilon+\lambda) /(\Gamma(\psi) \Gamma(e-\epsilon) \Gamma(\lambda))},
$$

and

$$
\widetilde{\mathcal{I}}_{4}=\frac{1}{N} \sum_{i=1}^{N} \frac{u_{i}^{a_{i}-1}\left(1-u_{i}\right)^{b_{i}-1} v_{i}^{c_{i}-1}\left(1-v_{i}\right)^{d_{i}-1}\left(u_{i}-v_{i}\right)^{e} / g\left(a_{i}, b_{i}, c_{i}, d_{i}, e\right)}{z\left(u_{i}, v_{i}\right) v_{i}^{\psi-1}\left(u_{i}-v_{i}\right)^{e-\epsilon-1}\left(1-u_{i}\right)^{\lambda-1} \Gamma(\psi+e-\epsilon+\lambda) /(\Gamma(\psi) \Gamma(e-\epsilon) \Gamma(\lambda))},
$$


where $\left(\left(u_{1}, v_{1}\right), \ldots,\left(u_{N}, v_{N}\right)\right)$ is an i.i.d. sample from $\mathcal{D}(\psi, e-\epsilon, \lambda)$ and the $\left(a_{i}, b_{i}, c_{i}, d_{i}\right)$ 's constitute the MCMC sample from the posterior distribution of $(a, b, c, d)$ given $(\mathbf{x}, \mathbf{y}) .^{8}$

The Bayes estimate of $\mathcal{I}$ is therefore approximated by the ratio of both importance sampling Bayes estimates, i.e. $\widetilde{\mathcal{I}}_{3} / \widetilde{\mathcal{I}}_{4}$.

The corresponding estimations of $\mathcal{I}_{1}, \mathcal{I}_{2}, \mathcal{I}_{3}, \mathcal{I}_{4}$ and $\mathcal{I}$ are given in Table 4, based on 200, 000 MCMC iterations. For $e=0.1$ and $e=0.05$, we used the last 190,000 MCMC iterations in the summation and $\epsilon=0.01$. For $e=0.01$, we used only the last 150,000 MCMC iterations in the summation and $\epsilon=0.001$. The estimates of $\mathcal{I}_{1}, \mathcal{I}_{2}$ and $\mathcal{I}$ are very stable with respect to the variations of $e$ even if $\mathcal{I}_{3}$ and $\mathcal{I}_{4}$ diverge when $e$ goes to zero.

Recall that the objective probability is 0.5 . As shown in Table 2, the average subjective probability which is given by $\mathcal{I}_{1}$ is around 0.37 . The average subjective probability weighted by the risk tolerance, which is given by $\mathcal{I}_{2}$, is much lower than $\mathcal{I}_{1}$ and is around 0.3 . As far as the link between optimism and risk aversion is concerned, these results indicate the presence of a positive correlation between optimism and risk aversion: more risk-averse individuals are more optimistic.

\section{Conclusion}

In this paper, we have adopted a Bayesian estimation approach in order to analyze the link between risk aversion and optimism and to estimate the risk tolerance weighted average of subjective probabilities (which represents the consensus probability of the group) as well as the unweighted average. In the analyzed survey, while the objective probability is 0.5 , the estimated (equally-weighted) average subjective probability reveals to be around 0.4 and the estimated average subjective probability weighted by the risk tolerance reveals to be around 0.3. It appears then, on our sample, that the group is pessimistic and more pessimistic than the average individual.

We do not pretend that 0.3 represents the collective subjective probability in a general setting, nor that $\frac{0.4-0.5}{0.5}=-0.2$ represents the average level of optimism in all risk-taking situations in a $[-1,1]$ scale. We only claim that the difference between the unweighted and the risk tolerance weighted averages of subjective probabilities is substantial enough to conclude to a behavioral correlation between optimism and risk aversion. Analogously, the difference between the objective probability and the unweighted average of subjective probabilities is substantial enough to conclude to a behavioral bias toward pessimism at the individual level. As a consequence there should be a bias toward pessimism at the collective level. The impact on the equilibrium characteristics should then be towards an increase of the risk premium and a decrease of the risk-free rate, which is interesting with regard to the risk premium and risk free rate puzzles.

To identify the origin of the elicited pessimism and correlation between optimism and risk aversion is a delicate question. The observed pessimism might result from an individual learning process, where individuals overestimate bad experiences (see Taylor, 1991) and the intensity of this effect might be related to the individual level of risk aversion. Besides, people seem to be disappointment averse in their choices (Bell, 1985). Disappointment avoidance may reflect a self deception mechanism designed to protect self esteem about decision making ability, i.e. a calculated avoidance of unpleasant future feelings (Joseph et al., 1996, Gollier and Muerman, 2006). The elicited pessimism could be interpreted as defensive pessimism, an anticipatory strategy that

\footnotetext{
${ }^{8}$ It may sound surprising to mix $\left(u_{i}, v_{i}\right)$ 's simulated from the importance function with $\left(a_{i}, b_{i}, c_{i}, d_{i}\right)$ 's simulated from the posterior distribution, but the average $\widetilde{\mathcal{I}_{3}}$ (resp. $\tilde{\mathcal{I}}_{4}$ ) is converging to the double expectations $\mathbb{E}\{\mathbb{E}[\pi / z(\pi, p)] \mid(\mathbf{x}, \mathbf{y})\}($ resp. $\mathbb{E}\{\mathbb{E}[1 / z(\pi, p)] \mid(\mathbf{x}, \mathbf{y})\})$.
} 
involves setting defensively low expectations prior to entering a situation so as to defend against loss of self esteem in the event of failure and here again the intensity of this effect might be related to the individual level of risk aversion. This would require a deeper analysis of the process of belief formation.

\section{References}

[1] Abel, A., 1989. Asset prices under heterogeneous beliefs: Implications for the equity premium. Mimeo, University of Pennsylvania.

[2] Barsky R., Juster F.T., Kimball M.S. and Shapiro M.D., 1997. Preference parameters and behavioural heterogeneity. An experimental approach in the health and retirement study. The Quarterly Journal of Economics,112, 537-80.

[3] Bell, D. E., 1985. Disappointment in decision making under uncertainty. Operations Research, $33(1), 1-27$

[4] Ben Mansour, S., Jouini, E. and C. Napp, 2006. Is there a "pessimistic" bias in individual beliefs? Evidence from a simple survey. Theory and Decision, 2006, 61(4), 345-362.

[5] Binswanger, H.P, 1980. Attitudes toward risk: Experimental measurement in rural India. American Journal of Agricultural Economics, 62 (3), 395-407.

[6] Binswanger, H.P, 1981. Attitudes toward risk: Theoretical implications of an experiment in rural India. Economic Journal , 91, 867-890.

[7] Calvet, L., Grandmont, J.-M., and I. Lemaire, 2002. Aggregation of heterogeneous beliefs and asset pricing in complete financial markets. Working Paper.

[8] Cohn R.A., Lewellen W.G, Lease R.C and Schlarbaum G.G, 1975. Individual financial risk aversion and investment portfolio composition. Journal of Finance, 30, 605-620.

[9] Detemple, J. and S. Murthy, 1994. Intertemporal asset pricing with heterogeneous beliefs. Journal of Economic Theory, 62, 294-320.

[10] Donkers, B., Melenberg, B. and Van Soest, A., 2001. Estimating risk attitudes using lotteries: a large sample approach. Journal of Risk and Uncertainty, 22 (2), 165-195.

[11] Dreman, D. N. and M. Berry, 1995. Analyst forecasting errors and their implications for security analysts. Financial Analysts Journal 51, 30-40.

[12] Eisenhauer, J.G. and M. Halek, 2001. Demography of risk aversion. The Journal of Risk and Insurance, 68, 1, 1-24.

[13] Eisenhauer, J.G. and L.Ventura, 2003. Survey measures of risk aversion and prudence. Applied Economics, 35, 1477-1484.

[14] Felton, J., Gibson, B. and D.M. Sanbonmatsu, 2003. Preference for Risk in Investing as a Function of Trait Optimism and Gender, Journal of Behavioral Finance, 33-40. 
[15] Francis, J. and D. Philbrick, 1993. Analysts' decisions as products of a multi-task environment. Journal of Accounting Research 31, 216-230.

[16] Fried, D. and D. Givoly, 1982. Financial Analysts' Forecasts of Earnings: A Better Surrogate for Earnings Expectations. Journal of Accounting and Economics 4, 85-107

[17] Giordani, P. and P. Söderlind, 2005. Is there evidence of pessimism and doubt in subjective distributions? Implications for the risk premium puzzle. Journal of Economic Dynamics and Control, 30(6), 1027-1043.

[18] Gollier, C., 2003. Who Should we Believe? Collective risk-taking decisions with heterogeneous beliefs. IDEI Working Paper, n. 201, 2003.

[19] Gollier, C. and A. Muerman, 2006. Optimal choice and beliefs with ex Ante savoring and Ex Post disappointment. IDEI working Paper, 201.

[20] Guiso, L. and Paiella, M., 2001. Risk aversion, wealth and background risk. Discussion paper 2728, Center of Economic Policy Research, March.

[21] Hariharan G., Chapman K.S and Domian D.L., 2000. Risk tolerance and asset allocation for investors nearing retirement. Financial Services Review, 9, 159-170

[22] Hartog J., Carbonell A.F., and Jonker N., 2002. Linking measured risk aversion to individual characteristics. Kyklos, 55, 3-26

[23] Jianakoplos, N. A. and Bernasek, A., 1998. Are women more risk averse? Economic Inquiry, $36,620-30$.

[24] Joseph, R., Larrick, R.P, Steele, C.M and Nisbett, R.E., 1996. Protecting the self from the negative consequences of risky decisions. Journal of Personality and Social Psychology, 62, $26-37$

[25] Jouini, E. and Napp, C., 2006. Heterogenous beliefs and asset pricing in discrete time: an analysis of pessimism and doubt. Journal of Economic Dynamics and Control, 30, 1233-1260.

[26] Jouini, E. and Napp, C., 2007. Consensus consumer and intertemporal asset pricing with heterogeneous beliefs. Review of Economic Studies. Forthcoming.

[27] Kang, S., J. O'Brien and K. Sivaramakrishnan, 1994. Analysts' interim earnings forecasts: Evidence on the forecasting process. Journal of Accounting Research 32, 103-112.

[28] Li, T., 2006. Heterogeneous beliefs, asset prices, and volatility in a pure exchange economy, Journal of Economic Dynamics and Control, In Press.

[29] Mc Inish, T.H., 1982. Individual investors and risk-taking. Journal of Economic Psychology, $2,125-136$.

[30] Mehra, R., and E. Prescott, 1985. The equity premium: A puzzle. Journal of Monetary Economics, 15, 145-162.

[31] Morin, R. A. and Suarez, A. F., 1983. Risk aversion revisited. The Journal of Finance, 38(4), 1201-1216. 
[32] O'Brien, P. C., 1988. Analysts Forecasts as Earnings Expectations. Journal of Accounting and Economics 10, 53-88.

[33] Palsson A.M, 1996. Does the degree of risk aversion vary with household characteristics? Journal of Economic Psychology, 17, 771-787.

[34] Powell, M. and Ansic, D., 1997. Gender differences in risk behaviour in financial decision making: An experimental analysis. Journal of Economic Psychology, 18 (6).

[35] Riley W.B and Chow K.V, 1992. Asset allocation and individual risk aversion. Financial Analysts Journal, 48, 32-37.

[36] Robert, C. P., 2001. The Bayesian Choice, Springer-Verlag, New York.

[37] Robert, C.P. and Casella, G., 2004. Monte Carlo Statistical Methods, Springer-Verlag, New York.

[38] Rubinstein, M., 1976. The Strong Case for the Generalized Logarithmic Utility Model as the Premier Model of Financial Markets. Journal of Finance, 31, 551-571.

[39] Schooley D.K and Worden D.D, 1996. Risk aversion measures: comparing attitudes and asset allocation. Financial Services Review ,5, 87-99.

[40] Taylor, S., 1991. Asymmetric effects of positive and negative events: the MobilizationMinimization Hypothesis. Psychological Bulletin, 110 (1), 67-85.

[41] Wang, H. and Hanna, S., 1997. Does risk tolerance decrease with age? Financial Counseling and Planning, 8(2), 27-31.

[42] Weil, P., 1989. The equity premium puzzle and the risk-free rate puzzle. Journal of Monetary Economics, 24, 401-421.

[43] Wenglert, L. and Rosen, A.S., 2000. Measuring optimism-pessimism from beliefs about future events. Personality and Individual Differences, 28, 717-728.

[44] Zapatero, F., 1998. Effects on Financial Innovations on Market volatility when Beliefs are Heterogeneous. Journal of Economic Dynamics and Control, 22, 597-626. 


\section{Appendix}

Let us first define

$$
f_{\mathscr{G}}(y \mid x)=\frac{1}{4}\left(f_{\mathscr{G}\left(\delta_{1} x, \delta_{1}\right)}(y)+f_{\mathscr{G}\left(\delta_{2} x, \delta_{2}\right)}(y)+f_{\mathscr{G}\left(\delta_{3} x, \delta_{3}\right)}(y)+f_{\mathscr{G}\left(\delta_{4} x, \delta_{4}\right)}(y)\right) .
$$

The hybrid MCMC algorithm we used in our simulation is described by the following steps:

Hybrid MCMC algorithm

- Initialization: Simulate $\left(a^{(0)}, b^{(0)}, c^{(0)}, d^{(0)}\right)$ from $\otimes_{i=1}^{4} \mathscr{G} a(1,1)$

For $i \in\{1, \ldots, n\}$, simulate $\left(\pi_{i}^{(0)}, p_{i}^{(0)}\right)$ from

$$
\mathscr{B} e\left(x_{i}+a^{(0)}, 10-x_{i}+b^{(0)}\right) \otimes \mathscr{B} e_{] 0, \pi_{i}^{(0)}[}\left(y_{i}+c^{(0)}, 100-y_{i}+d^{(0)}\right)
$$

(truncated Beta distribution)

- For $t=1, \ldots$ :

1) For $i \in\{1, \ldots, n\}$, simulate $\left(\tilde{\pi}_{i}, \tilde{p}_{i}\right)$ from

$$
\mathscr{B} e\left(x_{i}+a^{(t-1)}, 10-x_{i}+b^{(t-1)}\right) \otimes \mathscr{B} e_{] 0, \pi_{i}^{(t)}[}\left(y_{i}+c^{(t-1)}, 100-y_{i}+d^{(t-1)}\right)
$$

and take

$$
\left(\pi_{i}^{(t)}, p_{i}^{(t)}\right)=\left\{\begin{array}{lll}
\left(\tilde{\pi}_{i}, \tilde{p}_{i}\right) & \text { with probability } & \rho_{i}^{(\pi, p)} \\
\left(\pi_{i}^{(t-1)}, p_{i}^{(t-1)}\right) & \text { with probability } & 1-\rho_{i}^{(\pi, p)}
\end{array}\right.
$$

where

$$
\rho_{i}^{(\pi, p)}=\min \left(\frac{\left(\tilde{\pi}_{i}-\tilde{p}_{i}\right)^{e^{(t-1)}} F_{\mathscr{B} e\left(c^{(t-1)}+y_{i}, d^{(t-1)}+100-y_{i}, 0,1\right)}\left(\tilde{\pi}_{i}\right)}{\left(\pi_{i}^{(t-1)}-p_{i}^{(t-1)}\right)^{e^{(t-1)}} F_{\mathscr{B} e\left(c^{(t-1)}+y_{i}, d^{(t-1)}+100-y_{i}, 0,1\right)}\left(\pi_{i}^{(t-1)}\right)}, 1\right)
$$

2) Simulate $\tilde{a}$ from $f_{\mathscr{G}}\left(\cdot \mid a^{(t-1)}\right)$ and take

$$
a^{(t)}=\left\{\begin{array}{lll}
\tilde{a} & \text { with probability } & \rho^{a}=\min \left(\frac{f_{a}(\tilde{a} \mid \ldots) / f_{\mathscr{G}}\left(\tilde{a} \mid a^{(t-1)}\right)}{f_{a}\left(a^{(t-1)} \mid \ldots\right) / f_{\mathscr{G}}\left(a^{(t-1)} \mid \tilde{a}\right)}, 1\right) \\
a^{(t-1)} & \text { with probability } \quad 1-\rho^{a}
\end{array}\right.
$$

3) Simulate $\tilde{b}$ from $f_{\mathscr{G}}\left(\cdot \mid b^{(t-1)}\right)$ and take

$$
b^{(t)}=\left\{\begin{array}{lll} 
& \text { with probability } & \rho^{b}=\min \left(\frac{f_{b}(\tilde{b} \mid \ldots) / f_{\mathscr{G}}\left(\tilde{b} \mid b^{(t-1)}\right)}{f_{b}\left(b^{(t-1)} \mid \ldots\right) / f_{\mathscr{G}}\left(b^{(t-1)} \mid \tilde{b}\right)}, 1\right. \\
b^{(t-1)} & \text { with probability } & 1-\rho^{b}
\end{array}\right)
$$


4) Simulate $\tilde{c}$ from $f_{\mathscr{G}}\left(\cdot \mid c^{(t-1)}\right)$ and take

$$
c^{(t)}=\left\{\begin{array}{lll}
\tilde{c} & \text { with probability } & \rho^{c}=\min \left(\frac{f_{c}(\tilde{c} \mid \ldots) / f_{\mathscr{G}}\left(\tilde{c} \mid c^{(t-1)}\right)}{f_{c}\left(c^{(t-1)} \mid \ldots\right) / f_{\mathscr{G}}\left(c^{(t-1)} \mid \tilde{c}\right)}, 1\right) \\
c^{(t-1)} & \text { with probability } & 1-\rho^{c}
\end{array}\right.
$$

5) Simulate $\tilde{d}$ from $f_{\mathscr{G}}\left(\cdot \mid d^{(t-1)}\right)$ and take

$$
d^{(t)}=\left\{\begin{array}{lll}
\tilde{d} & \text { with probability } & \rho^{d}=\min \left(\frac{f_{d}(\tilde{d} \mid \ldots) / f_{\mathscr{G}}\left(\tilde{d} \mid d^{(t-1)}\right)}{f_{d}\left(d^{(t-1)} \mid \ldots\right) / f_{\mathscr{G}}\left(d^{(t-1)} \mid \tilde{d}\right)}, 1\right) \\
d^{(t-1)} & \text { with probability } \quad 1-\rho^{d}
\end{array}\right.
$$

6) Simulate $\tilde{e}$ from $f_{\mathscr{G}}\left(\cdot \mid e^{(t-1)}\right)$ and take

$$
e^{(t)}=\left\{\begin{array}{lll}
\tilde{e} & \text { with probability } & \rho^{e}=\min \left(\frac{f_{e}(\tilde{e} \mid \ldots) / f_{\mathscr{G}}\left(\tilde{e} \mid e^{(t-1)}\right)}{f_{e}\left(e^{(t-1)} \mid \ldots\right) / f_{\mathscr{G}}\left(e^{(t-1)} \mid \tilde{e}\right)}, 1\right. \\
e^{(t-1)} & \text { with probability } \quad 1-\rho^{e}
\end{array}\right)
$$

After some experimenting, we found that the scales $\delta_{1}=1, \delta_{2}=10, \delta_{3}=100$ and $\delta_{4}=1000$ ensure a good behavior of the hybrid MCMC algorithm. 


\begin{tabular}{r|r|}
$c$ & $\mathrm{E}\left[O A_{i} * w_{i} \mid i \in c\right]$ \\
\hline 1 & 515.8272 \\
2 & 703.077 \\
3 & 608.1076 \\
4 & 951.7348 \\
5 & 758.1568 \\
6 & 1218.167 \\
7 & 1206.866
\end{tabular}

Table 1: This table reports for each class $c$ of relative risk aversion the average level over the individuals in that class of the product of absolute risk aversion (under the objective probability, $O A_{i}$ ) by the mean of the income band $w_{i}$. Kendall's Tau is equal to 0.71, Spearman's Rho is equal to 0.89 and Pearson's correlation coefficient is equal to 0.89 . There is then a positive relationship between the elicited levels of absolute and relative risk aversion.

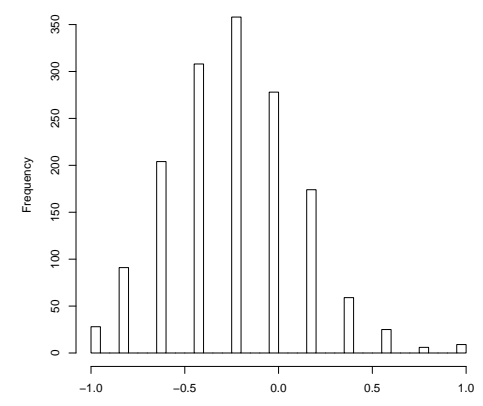

Figure 1: Distribution of pessimism. This figure represents the distribution of "pessimism" across our sample. A value of -1 (resp. 1) corresponds to people who think that they will never (resp. always) win at the head or tail game. The value of -0.1, which is endowed with the largest frequency of individuals, corresponds to people who think that they will win (or head will occur) 4 times out of ten. 


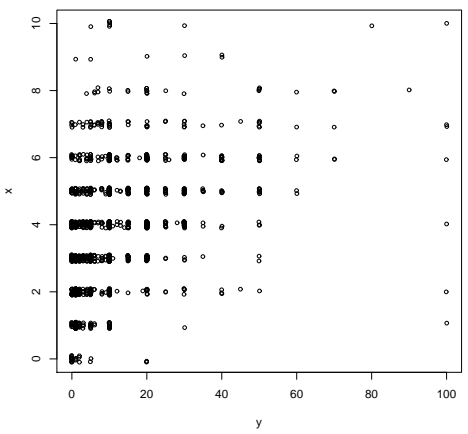

Figure 2: JITTER representation of the dataset. This figure reports on the horizontal axis the maximum price individuals are willing to pay in the game with ten draws and on the vertical axis the number of times they think that they will win.

\begin{tabular}{c|c|}
$x_{i}$ & $\mathrm{E}\left[C A_{i} \mid x_{i}\right]$ \\
\hline 1 & 0.7732720 \\
2 & 0.8224625 \\
3 & 0.7881803 \\
4 & 0.8831758 \\
5 & 1.1054212 \\
6 & 0.9278274 \\
7 & 1.4897416 \\
8 & 1.0668114 \\
9 & 5.1140936
\end{tabular}

Table 2: Average level of corrected risk aversion for a given level of $x$ (number of times the individual thinks he is going to win). Kendall's Tau is equal to 0.78 and Spearman's Rho is equal to 0.9 which indicates a positive link between optimism and corrected risk aversion.

\begin{tabular}{l|r|r|r|r}
$e$ & $\hat{a}$ & $\hat{b}$ & $\hat{c}$ & $\hat{d}$ \\
\hline 0.1 & 4.6305 & 8.5007 & 0.7207 & 3.1777 \\
0.05 & 4.5005 & 8.2155 & 0.7142 & 3.2730 \\
0.01 & 4.7623 & 8.5973 & 0.7283 & 3.6653
\end{tabular}

Table 3: MCMC estimates of $a, b, c, d$ (empirical means over the last 190,000 points in the MCMC sample out of 200,000 iterations). 

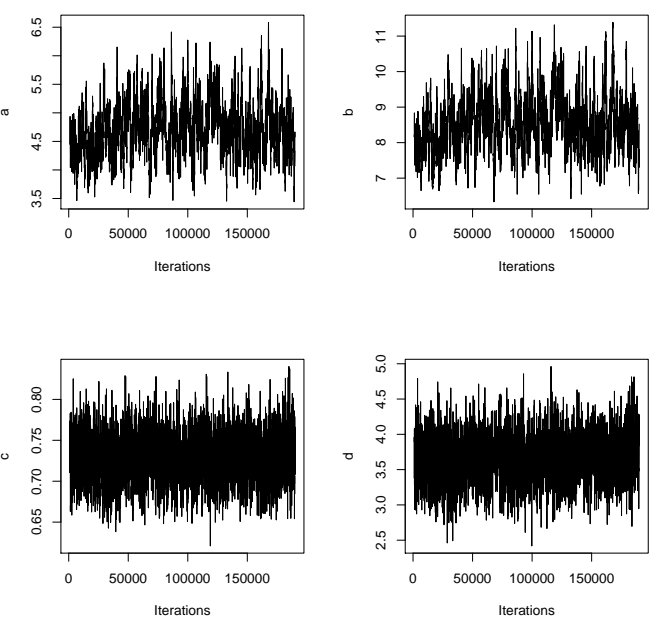

Figure 3: $e=0.01$ : evolution of the hybrid MCMC sample over 150,000 iterations
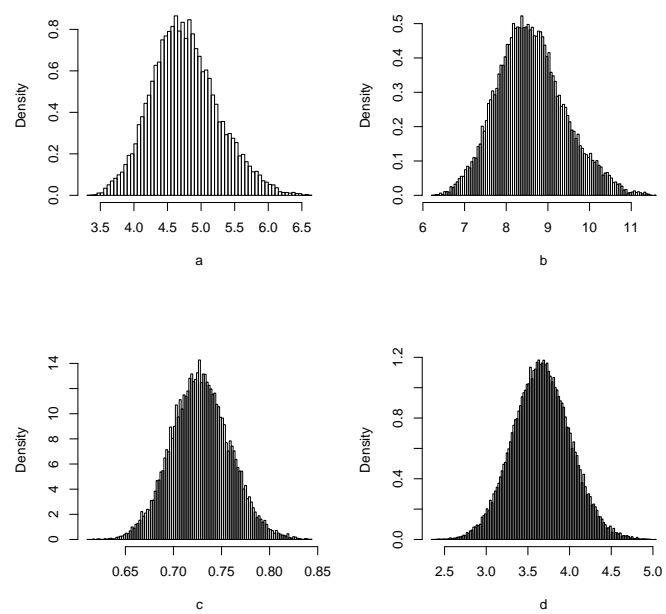

Figure 4: $e=0.01$ : histograms of the last 150,000 MCMC iterations

\begin{tabular}{l|r|r|r|r|r}
$e$ & $I_{1}$ & $I_{2}$ & $I_{3}$ & $I_{4}$ & $I$ \\
\hline 0.1 & 0.3770 & 0.1161 & 1.2458 & 4.1281 & 0.3018 \\
0.05 & 0.3755 & 0.1166 & 3.4904 & 11.7605 & 0.2968 \\
0.01 & 0.3735 & 0.1153 & 22.1699 & 74.6999 & 0.2967
\end{tabular}

Table 4: MCMC estimates of $\mathcal{I}_{1}, \mathcal{I}_{2}, \mathcal{I}_{3}, \mathcal{I}_{4}$ and $\mathcal{I}$ based on 200, 000 iterations. It is immediate to see that $\mathcal{I}_{3}$ and $\mathcal{I}_{4}$ diverge when $e$ goes to zero while their ratio $\mathcal{I}$ remains stable. 